2 DR MOHD HAFIZ ARZMI (Orcid ID : 0000-0002-9470-6412)

5 Article type : Special Issue Article

\title{
8 REVIEW ARTICLE
}

9 Title: Polymicrobial interactions of Candida albicans and its role in oral carcinogenesis

\section{Running title: Candida albicans and carcinogenesis}

13 Mohd Hafiz Arzmi ${ }^{1,2 *}$, Stuart Dashper ${ }^{1}$ and Michael McCullough ${ }^{1 *}$

$14{ }^{1}$ Melbourne Dental School, The University of Melbourne, Victoria, Australia

15 2Department of Fundamental Dental and Medical Sciences, Kulliyyah of Dentistry, International

16 Islamic University Malaysia, Kuantan, Pahang, Malaysia

18 Corresponding author:

19 Mohd Hafiz Arzmi (hafizarzmi@iium.edu.my)

20 Address: Department of Fundamental Dental and Medical Sciences, Kulliyyah of Dentistry, 21 International Islamic University Malaysia, Kuantan, Pahang, Malaysia

22 Telephone: +60132601445

\section{KEYWORDS}

25 Candida albicans, oral microbiome, polymicrobial interactions, oral carcinogenesis

\section{ABSTRACT}

This is the author manuscript accepted for publication and has undergone full peer review but has not been through the copyediting, typesetting, pagination and proofreading process, which may lead to differences between this version and the Version of Record. Please cite this article as doi: $\underline{10.1111 / J O P .12905}$

This article is protected by copyright. All rights reserved 
The oral microbiome is composed of microorganisms residing in the oral cavity, which are

2 critical components of health and disease. Disruption of the oral microbiome has been proven to

3 influence the course of oral diseases, especially amongst immunocompromised patients. Oral

4 microbiome is comprised of inter-kingdom microorganisms, including yeasts such as Candida

5 albicans, bacteria, archaea, and viruses. These microorganisms can interact synergistically, 6 mutualistically, and antagonistically, wherein the sum of these interactions dictates the composition 7 of the oral microbiome. For instance, polymicrobial interactions can improve the ability of $C$. 8 albicans to form biofilm, which subsequently increases the colonisation of oral mucosa by the yeast.

9 Polymicrobial interactions of $C$. albicans with other members of the oral microbiome have been reported to enhance the malignant phenotype of oral cancer cells, such as the attachment to extracellular matrix molecules (ECM) and epithelial-mesenchymal transition (EMT). Polymicrobial interactions may also exacerbate an inflammatory response in oral epithelial cells, which may play a role in carcinogenesis. This review focuses on the role of polymicrobial interactions between $C$. albicans and other oral microorganisms, including its role in promoting oral carcinogenesis.

Introduction

Oral cancer is one of the ten most prevalent cancers reported across the globe with more than $90 \%$ of mouth neoplasms identified as squamous cell carcinoma (1). With estimation of oral cancer incidences at approximately 275,000 cases, two-thirds of these are reported in developing countries (2). The risk factors that lead to oral squamous cell carcinoma (OSCC) include tobacco smoking, heavy alcohol consumption, poor oral hygiene, unhealthy diets, and microbial infection (3).

Microbial infections by yeast, bacteria, and viruses appear to have a causal role in oral cancer $(1,3)$. Candida spp. has been reported to be the most frequent fungal microbiome (mycobiome) isolated from the oral cavity, followed by Cladosporium, Aureobasidium, Saccharomycetales, Aspergillus, Fusarium, and Cryptococcus (4). Candida spp. may reflect a secondary infection of a pre-existing altered epithelium when linked with dysplasia. Oral yeast carriage has also been found to correlate with the presence of oral epithelial dysplasia (OED), which supports the function of microbial infection in oral carcinogenesis (5). Despite the numerous clinical and experimental conclusions describing the correlation between $C$. albicans and malignant transformation, the exact role of polymicrobial interactions in $C$. albicans for the development noted in dysplastic changes remains unclear. 


\section{Intra-kingdom and inter-kingdom interactions of Candida albicans}

C. albicans has been reported to be involved in both intra- and inter-kingdom interactions (6). Intra-kingdom interaction refers to the communication of the same kingdom, such as the interaction between Candida albicans (yeast) and Candida parapsilosis (yeast), whereas inter-kingdom interaction reflects microbial communication between two or more kingdoms, such as the interaction between C. albicans (yeast) and Streptococcus spp. (bacteria). Interaction of Candida spp. with other oral microorganisms has been reported to occur via co-aggregation and co-adhesion (7). Coaggregation is the association of genetically distinct microorganisms, such as the interaction between $C$. albicans and $S$. mutans in planktonic phase. On the other hand, co-adhesion is the interaction between microorganisms in planktonic phase with those on the oral surface (8). The interactions in oral cavity occur in oral biofilm (plaque), hence signifying a potential role in pathogenesis (9).

Although $C$. albicans is the most significant Candida spp. in pathogenesis, the discovery of non-albicans Candida (NAC) species, such as C. dubliniensis, C. glabrata, and C. tropicalis, has increased awareness among medical practitioners (10). Surprisingly, the carriage of NAC can outnumber that of C. albicans in certain conditions, such as the location of isolation. Additionally, the interaction between C. albicans and C. krusei varied depending on the nutrients supplied during the experiments (6).

The inter-kingdom interaction between C. albicans and Streptococcus spp. has been reported. C. albicans has been reported to co-aggregate $S$. mutans in planktonic phase potentially due to the presence of cell surface proteins on the bacterium that interact with cell surface carbohydrate of the yeast (8) (Figure 1). Antigen I/II is an example of antigenic protein of $S$. mutans that is involved in adherence to bacterium (11). S. mutans produces polysaccharides that have a role in the development and maturation of oral polymicrobial biofilms (12). Such mutualistic interaction between C. albicans and $S$. mutans is significant for C. albicans to colonise the oral surfaces, especially the soft tissues that may lead to inflammation. Despite being proven in in vitro studies that mutualistic interaction between $C$. albicans and $S$. mutans occurs through adhesions (non-specific) and lectin-saccharide cell surface receptors (specific) binding (13), antagonistic correlation between the two species has been reported as well. The adhesion of $C$. albicans on acrylic sheets appears to decrease when co-cultured with $S$. mutans in Gibbons and Nygaard culture medium (14). As for the quorum-sensing molecule, Farnesol synthesised by $C$. albicans during biofilm formation disrupts the cell membrane of $S$. mutans (15). Hence, it is suggested that the inter-kingdom interaction between $C$. albicans and $S$. mutans is dependent on C. albicans strains.

Candida spp. and Actinomyces spp. have been found to co-aggregate in varying degrees. Prior research has shown that the co-aggregation of $C$. albicans with $A$. naeslundii is $C$. albicans strain- 
1 dependent (8) (Figure 1). A. naeslundii has been shown to form fimbriae that assist in the attachment

2 of bacteria to oral surfaces via cell-to-cell and cell-to-surface adhesion mechanisms (7). This

3 characteristic aids in the formation of polymicrobial biofilms that enhance the colonisation in the oral

4 cavity (7). Although co-aggregation of C. albicans and Actinomyces spp. has been reported (8), the

5 adhesion of $C$. albicans to polymethylmethacrylate (PMMA) in a flow cell system when grown in

6 TNMC buffer decreased when pre-cultured with A. naeslundii T14V-J1 (16). In addition, the

7 interaction of $\bar{A}$. naeslundii appeared to decrease the auto-aggregation in planktonic and the metabolic

8 rate in static biofilm of $C$. albicans in the presence of $S$. mutans $(6,8)$ (Figure 1). C. albicans with

9 low metabolic activity is more invasive and linked with disease; on the contrary, those with high activity are non-inyasive (17). Furthermore, low metabolic activity can reduce antifungal susceptibility of C. albicans towards amphotericin B, potentially due to minimal absorption of antifungal agents, thus affecting inactivation kinetics (18).

A study also showed that C. albicans antagonistically interacted with Pseudomonas spp., wherein the virulence factors include secretion of alkaline protease, exotoxins, endotoxins, and biofilm forming ability (19). P. aeruginosa can form dense biofilm on C. albicans and receive nutrients from candidal hyphae, which later kills the yeast (20). P. aeruginosa has been reported to inhibit the formation of $C$. albicans biofilm by synthesising anti-candidal agents, such as extracellular bacterial glycocalyx, pyrrolnitrin, and 3-oxo-C12 homoserine lactone (20).

The antagonistic interaction between $C$. albicans and Porphyromonas spp. has also been reported. Porphyromonas spp. is an anaerobic and black-pigmented Gram-negative bacterium, in which $P$. gingivalis has been among the major periodontal pathogens. $P$. gingivalis produces proteases that destroy haemolysin, haeme-sequestering proteins, collagens, immunoglobulins, and complements (21). $P$, gingivalis was reported to suppress adhesion of $C$. albicans to acrylic surfaces by inhibiting germ-tube formation of $C$. albicans. Despite the inhibition of germ-tube formation of $C$. albicans, this interaction enhances invasion of $P$. gingivalis on gingival epithelial tissue potentially due to the increased expression of FimA component of $P$. gingivalis that is responsible for the adhesion of bacteria to the oral surfaces (22).

\section{Polymicrobial biofilms of Candida albicans and oral microbiome}

Polymicrobial biofilm is defined as the community of microorganisms attached to oral surfaces and encapsulated within extracellular polysaccharides (EPS) of microbial and salivary origin (23). Cell-cell signalling mechanisms between the members of polymicrobial biofilm contribute to the successful colonisation of bacteria. 
Biofilms have been reported to increase the resistance of $C$. albicans to fluconazole, up to 1000-fold, thus increasing its pathogenicity towards the host (17). EPS encapsulates the work of

3 biofilms, making it a barrier that limits the penetration of antimicrobial agents, subsequently reducing

4 the susceptibility of the targeted microorganisms. Constriction of nutrients that occurs within biofilms at the basal region reduces microbial growth and metabolic rate, apart from altering the microbial cell surface composition (18). The metabolic activity of C. albicans is reported to increase in the presence of A. naeslundii in dual-cultured biofilms. Nevertheless, a decrease in metabolic activity was observed in tri-cultured biofilms, when compared to dual-cultured biofilms of C. albicans and A. naeslundii, suggesting that these microorganisms may interact in a metabolic manner (24). $C$. albicans with low metabolic activity is more invasive, which may be linked with oral carcinogenesis, while conversely, those with high activity are non-invasive (17). Slower growing microorganisms can represent 'persister cells'. Persister cells possess a resistant phenotype that minimises the susceptibility of polymicrobial community within biofilm to antimicrobial agents (25).

In addition, the biomass and the percentage surface colonisation of $C$. albicans is significantly reduced in polymicrobial biofilms of $C$. albicans, A. naeslundii, and $S$. mutans, in comparison to the biomass of $C$. albicans in mono-cultured biofilms when grown in flow cell environment (24) (Figure 2). This condition is due to the metabolic products of A. naeslundii that have been reported to both inhibit and stimulate the biofilm formation of $C$. albicans, depending on the experimental methods employed and the strains of C. albicans used (6).

\section{Effect of polymicrobial biofilms of Candida albicans and oral microbiome on normal and} cancerous oral epithelial cells phenotypes

Carcinoma refers to the ability of malignant cell to invade the underlying connective tissues, 24 and is followed by metastases to distant sites (26). These processes demand the alteration of cell-tocell and cell-to-extracellular matrix (ECM) interactions that involve adhesion molecules, such as collagen, laminin, fibronectin, and fibrinogen (27). Several studies have also revealed the distinct variation in the ability of normal oral epithelial cells and OSCC isolated cells to adhere to ECM components upon incubation with polymicrobial biofilm effluent, with cancerous cells increasing their adhesion to collagen IV and laminin I in a significant manner, when compared to mono-species biofilm effluent (24). The variability of adhesion for normal and cancerous cells to ECM components

31 is due to the alteration of cell integrins following interaction with biofilm effluent (27). Integrins have 32 been recognised as the largest family of cell adhesion molecules that consist of multiple combinations 33 of $\alpha$ - and $\beta$-subunits (28). Proteins synthesised by microorganisms, such as proteases from $C$. 34 albicans and Bcl-2 family proteins from bacteria, have been shown to induce alteration of integrins in 
1 epithelial cells (3). The $\alpha 1$-subunit of integrins that preferentially binds to collagen IV, and $\alpha 3-$ 2 subunit that functions as a receptor for laminins, have been reported to change during interaction with 3 the proteins of microorganisms (27). Subsequent trigger of a spectrum of signals involved in the 4 process of growth and proliferation may also promote oral carcinogenesis in a paracrine fashion (29).

Epithelial-mesenchymal transition (EMT) is a mechanism that alters cell-to-cell and cell-toECM interactions so as to allow the movement of cancerous cells to the surrounding environment. Nonetheless, inappropriate utilisation of EMT may occur in the formation of OSCC and metastasis of malignant cells (30). EMT can also be involved in increasing resistance of malignant cells to apoptosis regulator molecules (31). Vimentin and E-cadherin have been widely used as markers for EMT. The expression of vimentin induces changes in cell shape, motility, and adhesion of epithelial cells to mesenchyme; while cadherins mediate cell-to-cell binding that is critical in maintaining tissue structure and morphology (32). Functional loss of E-cadherin in epithelial cells has been considered as a marker for EMT during tumour progression (33). A previous study has reported that the expression of E-cadherin from normal epithelial cells increased after 24-h of incubation with monocultured biofilm effluent, which is likely to indicate adhesion, colonisation, internalisation, and potentially, invasion (24). Increment in E-cadherin expression appears to be a strategy of colonisation among C. albicans, A. naeslundii, and S. mutans to oral epithelial cells (34). Additionally, cadherins have been reported to form a route for the internalisation of $C$. albicans into epithelial cells during oral thrush $(34,35)$. Als3 protein synthesised by $C$. albicans has also been thought to mimic cadherin-cadherin binding, thus initiating the invasion of yeast to oral epithelial cells that may promote oral carcinogenesis (35).

Inflammation induced by pathogens has been shown to be involved in carcinogenesis (36). One factor that leads to inflammation is increase of pro-inflammatory cytokines due to microbial infection of oral mucosa (37). Cytokines are signalling molecules that regulate the differentiation, proliferation, and many other crucial functions in human inflammatory cells. Cytokine signals are received at the cell surface, not only as a single message, but also in complex, subtle, synergistic, and antagonistic combinations that coordinate processes, including stimulation of haematopoiesis, orchestration of directed leukocyte migration (chemokinesis), activation of various inflammatory cells, stimulation of lymphocyte development and maturation, as well as processes associated with immune response (38). Nonetheless, in certain circumstances, such as failure to resolve an injury, this condition may provoke excessive immune cell infiltration that may persistently generate cytokine. As

a result, the host may respond to persistent cytokine expression by enhancing cancer formation and 
1 progression (39). Cytokines that have been shown to be involved in inflammation include interleukin 2 (IL)-1 $\alpha$, IL-1 $\beta$, IL-6, IL-8, IL-18, TNF- $\alpha$, IFN- $\gamma$, and GM-CSF (40).

Previous studies have reported increase in cancerous inflammatory cytokines; IL-6 and IL-8, 4 from normal and cancerous oral cell lines upon incubation in mono-species and polymicrobial 5 biofilms of $C$. albicans, A. naeslundii, and S.mutans effluents (24). The increased expression of pro6 inflammatory cytokines, particularly IL-6 and IL-8, are significant in both inflammation and tumorigenesis of malignant cells. The increased expression of IL- 6 and IL-8 by oral cancer cells may

8 be due to proteins glycosylated with $\mathrm{N}$ - or O-linked mannosyl residues, $\beta$-glucans, and chitins on $C$.

9 albicans cell wall, as well as the expression of secreted aspartyl proteinases (SAPs) from yeast (41).

10 IL-6 has been proven to exert anti-apoptotic effect on malignant cells (42). The direct autocrine 11 tumour-promoting effects of IL-6 have been demonstrated in multiple myeloma by both increasing 12 proliferation and preventing apoptosis (43). It has also been shown to promote head and neck tumour 13 metastases by inducing EMT via the JAK-STAT3-SNAIL signalling pathway (33). This cytokine is 14 one of the main chemokines present in serum samples of head and neck cancer patients, wherein 15 elevated IL-6 levels can independently predict tumour recurrence, poor survival, and tumour 16 metastasis (44). IL-6 in serum has been found to be higher in patients with OSCC, when compared to controls, thus its proposal as an additional marker in early detection of oral cancer (45). This cytokine is a major mediator that links inflammation to cancers, along with TNF- $\alpha$ (46).

Meanwhile, the expression of IL-8 by human carcinoma cells has been reported to directly correlate with tumour vascularity and cancer progression. Research has shown that IL-8 can be produced by various types of tumours, both constitutively and in response to cytokines (47). This cytokine has been detected in surgical specimens, freshly cultured cell lines, as well as well-defined cell lines of head and neck squamous cell carcinoma (48). IL-8 stimulates a substantial number of physiopathological functions in various tumour cells, including prostate carcinoma, ovarian carcinoma, melanoma, and gastric carcinoma cell lines (49). The high expression of IL-8 in cancerous liver tissue has been associated with a higher frequency of portal vein, venous, and bile duct invasion in hepatocellular carcinoma patients with surgical resection, thus may be crucial in invasion and metastasis (50).

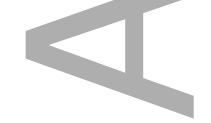

\section{Conclusion}

31 Polymicrobial interactions of $C$. albicans with oral microorganisms $A$. naeslundii and $S$. mutans appears to promote oral carcinogenesis by not only enhancing invasion of oral squamous cell 
1 carcinoma as demonstrated by increased adhesion to extra-cellular matrix proteins, but also by 2 increasing pro-inflammatory cytokine expression. This potentially may have profound clinical

3 implications, and further research to elucidate the exact mechanism is necessary.

The authors would like to acknowledge OHCRC, Melbourne Dental School, The University of Melbourne and Malaysia's Ministry of Education for funding (FRGS19-024-0632).

\section{Conflict of interest}

The authors declare no conflict of interest.

\section{References}

1. Pushalkar S, Mane SP, Ji X et al. Microbial diversity in saliva of oral squamous cell carcinoma. FEMS Immunol Med Microbiol 2011; 61: 269-77.

2. Ferlay J, Bray F, Pisani P, Parkin D. GLOBOCAN 2002: Cancer Incidence, Mortality and Prevalence Worldwide. IARC Cancer Base No. 5. version 2.0. Lyon: IARC Press; 2004.

3. Khajuria N, Metgud R. Role of bacteria in oral carcinogenesis. Indian J Dent 2015; 6: 37.

4. Ghannoum MA, Jurevic RJ, Mukherjee PK et al. Characterization of the oral fungal microbiome (mycobiome) in healthy individuals. PLoS Pathog 2010; 6: e1000713.

5. McCullough M, Jaber M, Barrett A, Bain L, Speight P, Porter S. Oral yeast carriage correlates with presence of oral epithelial dysplasia. Oral Oncol 2002; 38: 391-3.

6. Arzmi MH, Alnuaimi AD, Dashper S, Cirillo N, Reynolds EC, McCullough M. Polymicrobial biofilm formation by Candida albicans, Actinomyces naeslundii and Streptococcus mutans is Candida albicans strain and medium dependent. Med Mycol 2016; DOI: 10.1093/mmy/myw042.

7. Kolenbrander PE, Palmer RJ, Periasamy S, Jakubovics NS. Oral multispecies biofilm development and the key role of cell-cell distance. Nat Rev Microbiol 2010; 8: 471-480.

8. Arzmi MH, Dashper S, Catmull D, Cirillo N, Reynolds E, McCullough M. Co-aggregation of Candida albicans, Actinomyces naeslundii and Streptococcus mutans is Candida albicans strain dependent. FEMS Yeast Res 2015; DOI: 10.1093/femsyr/fov038. 
9. Tan K, Seers CA, Dashper SG et al. Treponema denticola and Porphyromonas gingivalis exhibit metabolic symbioses. PLOS Pathog 2014; DOI: 10.1371/journal.ppat.1003955.

10. Alnuaimi AD, Wiesenfeld D, O’Brien-Simpson NM, Reynolds EC, McCullough MJ. Oral Candida colonization in oral cancer patients and its relationship with traditional risk factors of oral cancer: A matched case-control study. Oral Oncol 2015; 51: 139-45.

11. Chuzeville S, Dramsi S, Madec J-Y, Haenni M, Payot S. Antigen I/II encoded by integrative and conjugative elements of Streptococcus agalactiae and role in biofilm formation. Microb Pathog 2015; 88: 1-9.

12. Koo H, Xiao J, Klein M, Jeon J. Exopolysaccharides produced by Streptococcus mutans glucosyltransferases modulate the establishment of microcolonies within multispecies biofilms. J Bacteriol 2010; 192: 3024-32.

13. Ledder RG, Timperley, AS, Friswell MK, Macfarlane S, McBain AJ. Coaggregation between and among human intestinal and oral bacteria. FEMS Microbiol Ecol 2008; 66: 630-6.

14. Barbieri DSAV, Vicente VA, Fraiz FC, Lavoranti OJ, Svidzinski TIE, Pinheiro RL. Analysis of the in vitro adherence of Streptococcus mutans and Candida albicans. Braz J Microbiol 2007; 38: 624-631.

15. Černáková L, Jordao L, Bujdáková H. Impact of farnesol and Corsodyl® on Candida albicans forming dual biofilm with Streptococcus mutans. Oral Dis 2018; 24: 1126-31.

16. Millsap KW, Bos R, Van Der Mei HC, Busscher HJ. Dot assay for determining adhesive interactions between yeasts and bacteria under controlled hydrodynamic conditions. $J$ Microbiol Methods 2000; 40: 225-32.

17. Tobudic S, Kratzer C, Lassnigg A, Presterl E. Antifungal susceptibility of Candida albicans in biofilms. Mycoses 2012; 55: 199-204.

18. Mah TF, O' Toole GA. Mechanisms of biofilm resistance to antimicrobial agents. Trends Microbiol 2001; 9: 34-39.

19. García-Contreras R, Pérez-Eretza B, Lira-Silva E et al. Gallium induces the production of virulence factors in Pseudomonas aeruginosa. Pathog Dis 2014; 70: 95-98.

20. Hogan DA, Kolter R. Pseudomonas-Candida interactions: an ecological role for virulence factors. Science 2002; 296: 2229-32.

21. Hajishengallis G, Darveau RP, Curtis MA. The keystone-pathogen hypothesis. Nat Rev Microbiol 2012; 10: 717.

22. Sztukowska MN, Dutton LC, Delaney C et al. Community Development between Porphyromonas gingivalis and Candida albicans Mediated by InlJ and Als3. mBio 2018; 9: e00202-00218.

This article is protected by copyright. All rights reserved 
23. O'donnell LE, Millhouse E, Sherry L et al. Polymicrobial Candida biofilms: friends and foe in the oral cavity. FEMS Yeast Res 2015; 15.

24. Arzmi MH, Cirillo N, Lenzo JC et al. Monospecies and polymicrobial biofilms differentially regulate the phenotype of genotype-specific oral cancer cells. Carcin 2018.

25. Cameron DR, Shan Y, Zalis EA, Isabella V, Lewis K. A genetic determinant of persister cell formation in bacterial pathogens. J Bacteriol 2018; 200: e00303-00318.

26. Clark AG, Vignjevic DM. Modes of cancer cell invasion and the role of the microenvironment. Curr Opin Cell Biol 2015; 36: 13-22.

27. Lyons A, Jones J. Cell adhesion molecules, the extracellular matrix and oral squamous carcinoma. Int J Oral Maxillofac Surg 2007; 36: 671-9.

28. Streuli CH. Integrins as architects of cell behavior. Mol Biol Cell 2016; 27: 2885-8.

29. Meurman JH. Infectious and dietary risk factors of oral cancer. Oral Oncol 2010; 46: 411-3. 6.

31. Brabletz T, Kalluri R, Nieto MA, Weinberg RA., EMT in cancer. Nat Rev Cancer 2018; 18 : 128.

32. Mendez MG, Kojima SI, Goldman RD. Vimentin induces changes in cell shape, motility, and adhesion during the epithelial to mesenchymal transition. FASEB J 2010; 24: 1838-51.

33. Yadav A, Kumar B, Datta J, Teknos TN, Kumar P. IL-6 promotes head and neck tumor metastasis by inducing epithelial-mesenchymal transition via the JAK-STAT3-SNAIL signaling pathway. Mol Cancer Res 2011; 9: 1658-67.

34. Delva E, Kowalczyk AP. Regulation of cadherin trafficking. Traffic 2009; 10: 259-67.

35. Phan QT, Fratti RA, Prasadarao NV, Edwards JE, Filler SG. N-cadherin mediates endocytosis of Candida albicans by endothelial cells. J Biol Chem 2005; 280: 10455-10461.

36. Dzutsev A, Goldszmid RS, Viaud S, Zitvogel L, Trinchieri G. The role of the microbiota in inflammation, carcinogenesis, and cancer therapy. Eur J Immunol 2015; 45: 17-31.

37. Fantini MC, Pallone F. Cytokines: from gut inflammation to colorectal cancer. Curr Drug Targets 2008; 9: 375-80.

38. Budhu A, Wang XW The role of cytokines in hepatocellular carcinoma. J Leukoc Biol 2006; 80: $1197-213$.

39. Roshani R, McCarthy F, Hagemann T. Inflammatory cytokines in human pancreatic cancer. Cancer Lett 2014; 345: 157-63.

40. Lippitz BE. Cytokine patterns in patients with cancer: a systematic review. Lancet Oncol 2013; 14: e218-e228. 
41. Dongari-Bagtzoglou A, Kashleva H. Candida albicans triggers interleukin-8 secretion by oral epithelial cells. Microb Pathog 2003; 34: 169-77.

42. Chen Y, Zhang F, Tsai Y et al. IL-6 signaling promotes DNA repair and prevents apoptosis in CD133+ stem-like cells of lung cancer after radiation. Radiat Oncol 2015; 10: 227.

43. De Simone V, Franze E, Ronchetti G et al. Th17-type cytokines, IL-6 and TNF- $\alpha$ synergistically activate STAT3 and NF-kB to promote colorectal cancer cell growth. Oncogene 2015; 34: 3493.

44. Duffy SA, Taylor JM, Terrell JE et al. Interleukin-6 predicts recurrence and survival among head and neck cancer patients. Cancer 2008; 113: 750-7.

45. Rao M, Ramesh A, Adapa S, Thomas B, Shetty J. Correlation of salivary levels of interleukin6 and albumin with oral squamous cell carcinoma. J Health Res Rev 2016; 3: 11.

46. Balkwill FR, Mantovani A. Cancer-related inflammation: common themes and therapeutic opportunities. Semin Cancer Biol 2012; 77: 33-40.

47. Kolář M, Szabo P, Dvořánková B et al. Upregulation of IL-6, IL-8 and CXCL-1 production in dermal fibroblasts by normal/malignant epithelial cells in vitro: Immunohistochemical and transcriptomic analyses. Biol Cell 2012; 104: 738-51.

48. Polanska $\mathrm{H}$, Raudenska M, Gumulec J et al. Clinical significance of head and neck squamous cell cancer biomarkers. Oral Oncol 2014; 50: 168-77.

49. Yin J, Zeng F, Wu N, Kang K, Yang Z, Yang H. Interleukin-8 promotes human ovarian cancer cell migration by epithelial-mesenchymal transition induction in vitro. Clin Transl Oncol 2015; 17: 365-370.

50. Bai ZF, Tai Y, Li W et al. Gankyrin activates IL-8 to promote hepatic metastasis of colorectal cancer. Cancer Res 2013; doi: 10.1158/0008-5472.can-12-4586.

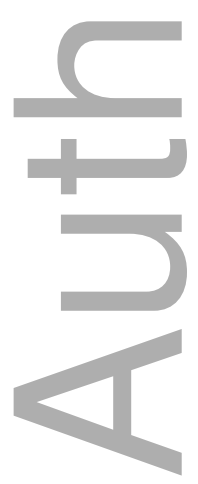




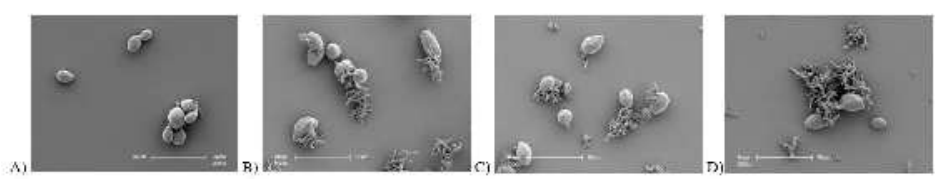

jop_12905_f1.jpg

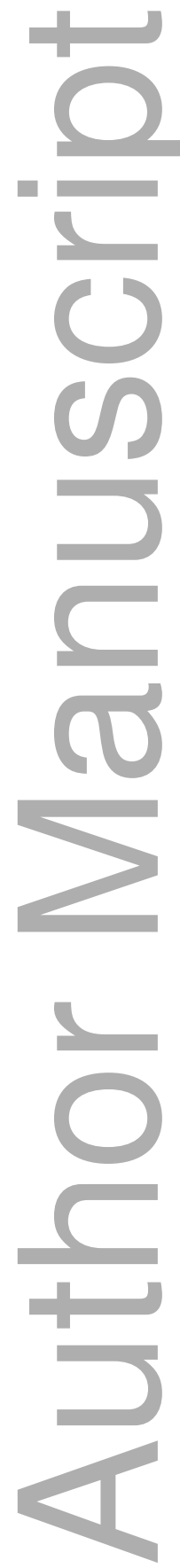

This article is protected by copyright. All rights reserved 


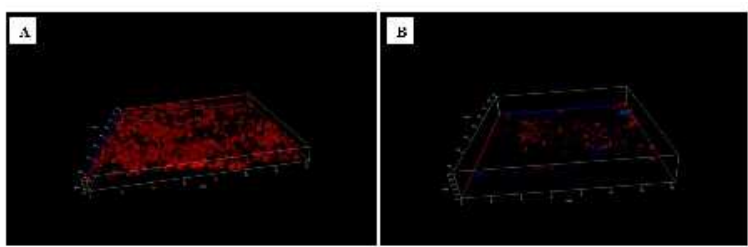

jop_12905_f2.jpg
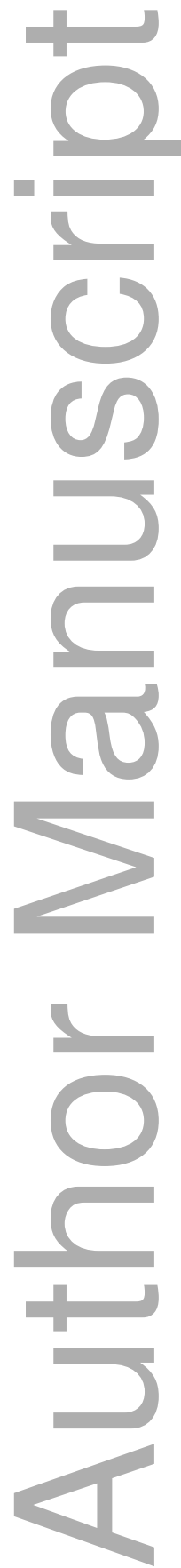


\section{University Library}

\section{- M M N E R VA A gateway to Melbourne's research publications}

Minerva Access is the Institutional Repository of The University of Melbourne

Author/s:

Arzmi, MH;Dashper, S;McCullough, M

Title:

Polymicrobial interactions of Candida albicans and its role in oral carcinogenesis

Date:

2019-08-01

Citation:

Arzmi, M. H., Dashper, S. \& McCullough, M. (2019). Polymicrobial interactions of Candida albicans and its role in oral carcinogenesis. JOURNAL OF ORAL PATHOLOGY \& MEDICINE, 48 (7), pp.546-551. https://doi.org/10.1111/jop.12905.

Persistent Link:

http://hdl.handle.net/11343/286040 